livraisons

d'Histoire

de l'Architecture

\section{Livraisons de l'histoire de l'architecture}

24 | 2012

Le phare et l'architecte

\title{
Maurice Durand et les phares de Vendée
}

Maurice Durand and the Lighthouses of Vendée

Maurice Durand und die Leuchttürme der Vendée

\section{Alain Delaval}

\section{OpenEdition}

\section{Journals}

Édition électronique

URL : http://journals.openedition.org/lha/101

DOI : 10.4000/lha.101

ISSN : 1960-5994

Éditeur

Association Livraisons d'histoire de l'architecture - LHA

Édition imprimée

Date de publication : 19 décembre 2012

Pagination : 149-158

ISSN : $1627-4970$

Référence électronique

Alain Delaval, « Maurice Durand et les phares de Vendée », Livraisons de l'histoire de l'architecture [En ligne], 24 | 2012, mis en ligne le 16 avril 2015, consulté le 07 mai 2019. URL : http:// journals.openedition.org/lha/101; DOI : 10.4000/lha.101

Ce document a été généré automatiquement le 7 mai 2019.

Tous droits réservés à l'Association LHA 


\title{
Maurice Durand et les phares de Vendée
}

\author{
Maurice Durand and the Lighthouses of Vendée \\ Maurice Durand und die Leuchttürme der Vendée
}

Alain Delaval

Nous remercions vivement notre collègue Olivier Liardet de nous avoir communiqué une abondante documentation pour cet article.

1 Le littoral du département de la Vendée est équipé, depuis le XIX siècle, d'un nombre important de phares et d'éléments de signalisation maritime, en raison des caractéristiques morphologiques de son profil côtier. La côte de Vendée peut en effet se diviser en trois zones principales: le secteur de la baie de Bourgneuf et de l'île de Noirmoutier, qui se rattache, géographiquement et sur le plan de la navigation maritime, à l'estuaire de la Loire ; la longue ligne dunaire du pays de Monts et du pays des Olonnes, ponctuellement interrompu par le plateau rocheux de Croix-de-Vie et l'embouchure de la Vie, fleuve côtier rejoint par son petit affluent le Jaunay, et, au large de cette côte, l'île d'Yeu ; la côte méridionale orientée au sud-ouest, entre Les Sables-d'Olonne et la baie de L'Aiguillon.

2 Cette côte fut balisée à partir des années 1830, c'est à dire dès que la France s'est engagée dans une politique de modernisation de sa signalisation maritime. Les plus anciens ouvrages de signalisation ont été construits sur les îles, au Pilier, au nord-ouest de Noirmoutier, en 1827, et à l'île d'Yeu, à la Petite Foule en 1830. Les autres phares de cette côte ont été construits progressivement dans la seconde moitié du XIX siècle.

3 À la fin de la deuxième guerre mondiale, en 1944, les troupes allemandes d'occupation ont détruit plusieurs phares. Le 25 août 1944, peu de temps avant leur repli devant l'avancée des armées alliées, les Allemands ont détruit le phare de la Petite Foule de l'île d'Yeu, construit en 1830, le phare de la pointe des Corbeaux également sur l'île d'Yeu, construit en 1862, et le phare de la pointe du Grouin-du-Cou, à la Tranche-sur-Mer, faisant face à l'île de Ré, qui avait remplacé en 1866 un fanal construit en 1831. Après la guerre, ces phares ont dû être reconstruits. C'est le service des Phares et balises qui fut chargé de ces 
opérations; la maîtrise d'œuvre fut assurée par les ingénieurs des ponts et chaussées, mais avec l'assistance de l'architecte sablais Maurice Durand.

\section{Maurice Durand, architecte vendéen}

4 Maurice Durand était profondément ancré dans le pays vendéen et particulièrement aux Sables-d'Olonne, où il est né, le 6 décembre 1884. Très tôt attiré par le métier d'architecte, il reçut sa première formation de 1900 à 1903 dans le cabinet de l'architecte Gaston Réchin à Angers, où il prépara le concours d'admission à l'École nationale et spéciale des Beaux-Arts de Paris, section Architecture, où il fut admis en 1905.

III. 1 : Maurice Durand, « Un phare à l'entrée du port de New-York »

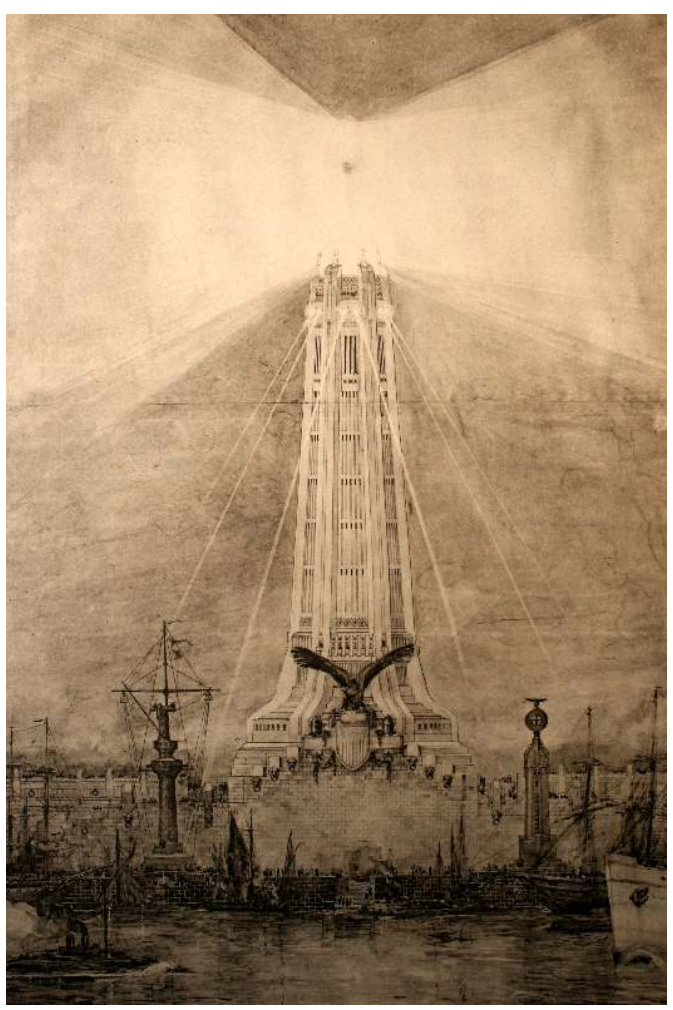

Concours pour le prix de reconnaissance des architectes américains, 1907, École nationale des Beaux-

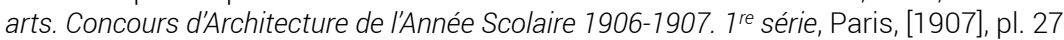

Cl. Olivier Liardet

5 Malgré une scolarité quelque peu tourmentée par des ennuis de santé mais aussi en raison d'un contentieux avec la direction de l'École en 1914, ayant été soupçonné de fraude sur sa situation de célibataire dans le cadre de la candidature au Prix de Rome, il se présenta avec succès à divers concours, notamment le Concours américain en 1907 avec un projet de " phare pour New-York »", et surtout le premier prix au concours Chenavard en 1909, avec un « pont monumental », et le prix Stillmann en $1912^{2}$.

Maurice Durand débuta sa carrière de bâtisseur dans sa ville natale, les Sables d'Olonne, en 1913, en construisant plusieurs maisons bourgeoises sur la promenade du Remblai, face à la mer. Maurice Durand est d'ailleurs essentiellement connu localement pour son activité auprès d'une clientèle privée à l'époque où la vieille cité portuaire, surtout habitée par une population vivant des ressources de la mer, se transformait en station 
balnéaire. Mais Maurice Durand ne fut pas que bâtisseur de demeures sablaises ; reçu à la Société centrale des architectes en 1920, il fut nommé Architecte de la ville des Sablesd'Olonne en 1921, puis Architecte départemental en 1924. Il produisit après la guerre de 1914-1918 plusieurs édifices publics aux Sables d'Olonne, notamment le casino de la Rudelière, l'église Saint-Pierre, la clinique Notre-Dame de France. Dans les années 1950-1960, Maurice Durand poursuivit son activité d'architecte municipal sur de nombreux chantiers: l'Hôtel de ville, le Grand Casino, l'aménagement paysager de la place du palais de Justice; à la fin de sa vie, il signa également la restauration de l'ancienne église Saint-Nicolas, sur la pointe rocheuse dominant l'entrée du port des Sables; il avait déjà marqué son intérêt pour l'architecture ancienne et les monuments religieux de sa région en acquérant en 1926 l'ancienne abbaye de Saint-Jean d'Orbestier, à quelques kilomètres des Sables d'Olonne, dont il restaura le logis abbatial. Maurice Durand est mort en $1978^{3}$.

\section{Des équipements reconstruits}

7 Après la deuxième guerre mondiale, Maurice Durand fut donc sollicité par le service des Phares et balises pour la reconstruction des phares détruits en 1944 par l'armée allemande, et donna les esquisses pour les formes architecturales à donner à ces ouvrages ${ }^{4}$. Les lignes très géométriques de ces phares, à la tour quadrangulaire à pans coupés ou à ressauts sont bâtis en fait selon des techniques traditionnelles, en maçonnerie de moellons de pierre, parfois, comme au Grouin-du-Cou, avec des matériaux de récupération de l'ancien phare détruit, et enduits au ciment revêtu d'une peinture au Silexore, seuls le socle et la plate-forme de la lanterne, ainsi que l'escalier intérieur étant montés en béton ${ }^{5}$. Les premiers furent les deux phares de l'île d'Yeu, celui de la Petite Foule, au centre de l'île, sur un plateau dominant la côte ouest, et celui de la pointe des Corbeaux, à l'extrémité sud-ouest. 
III. 2 : Île d'Yeu, phare de La Petite-Foule, face est

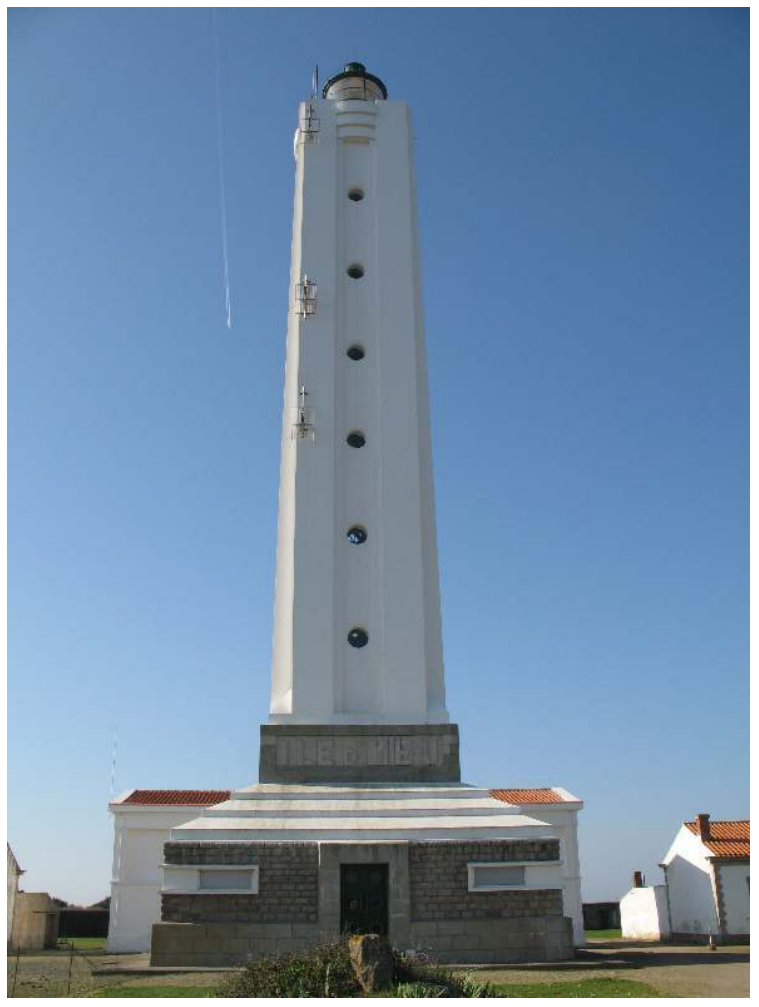

Cl. Alain Delaval

\section{3 : Île d'Yeu, phare des Corbeaux, face ouest}

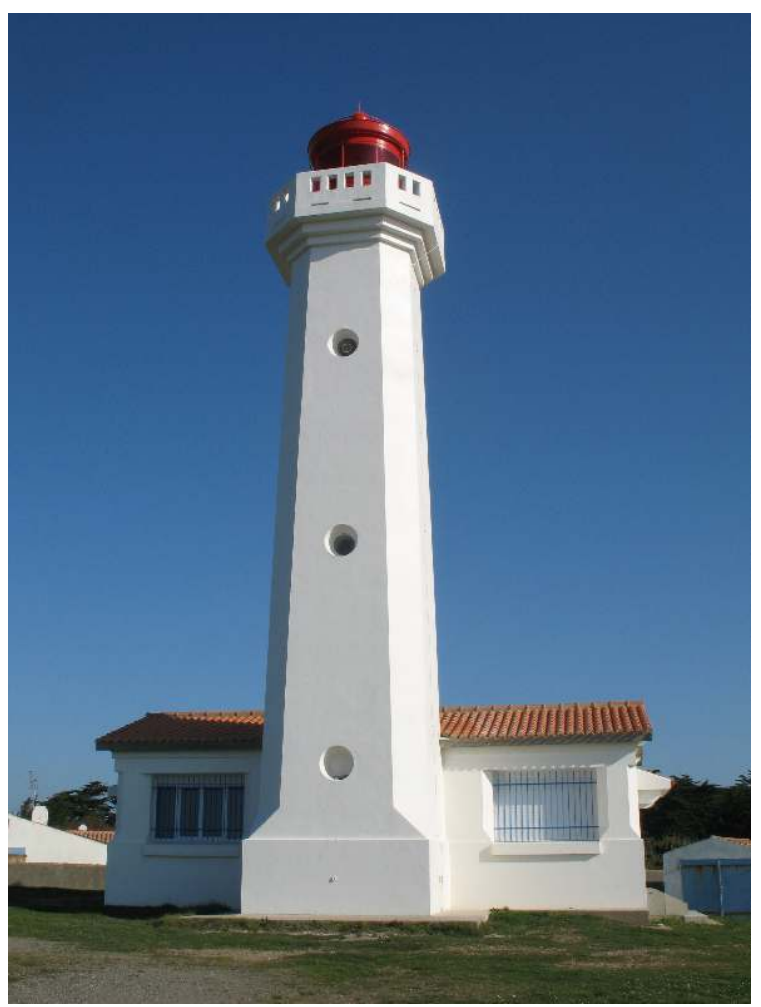

Cl. Alain Delaval 
8 Les deux phares furent construits en 1950; le phare des Corbeaux, de plus petites dimensions que le phare de la Petite Foule, élevé par Maurice Durand selon les indications de l'ingénieur Leconte, fut allumé l'année même ${ }^{6}$; le phare de la Petite Foule, appelé communément « le Grand Phare », fut terminé et mis en service en $1953^{7}$.

9 Cette même année, un autre phare détruit par l'armée allemande, le phare de la pointe du Grouin-du-Cou, à la Tranche-sur-Mer, était reconstruit encore une fois sur les plans de Maurice Durand ${ }^{8}$.

\section{4 : La Tranche-sur-Mer, phare du Grouin-du-Cou, le phare en cours de construction}

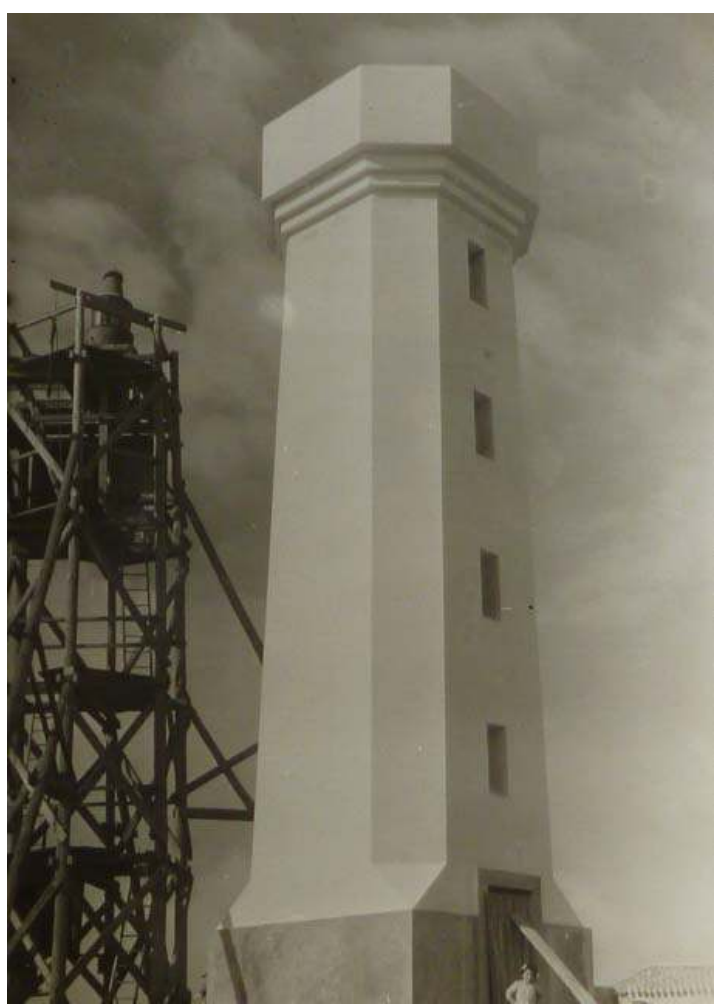

Photographie, 1953, Arch. dép. de la Vendée

$\mathrm{Cl}$. Alain Delaval 


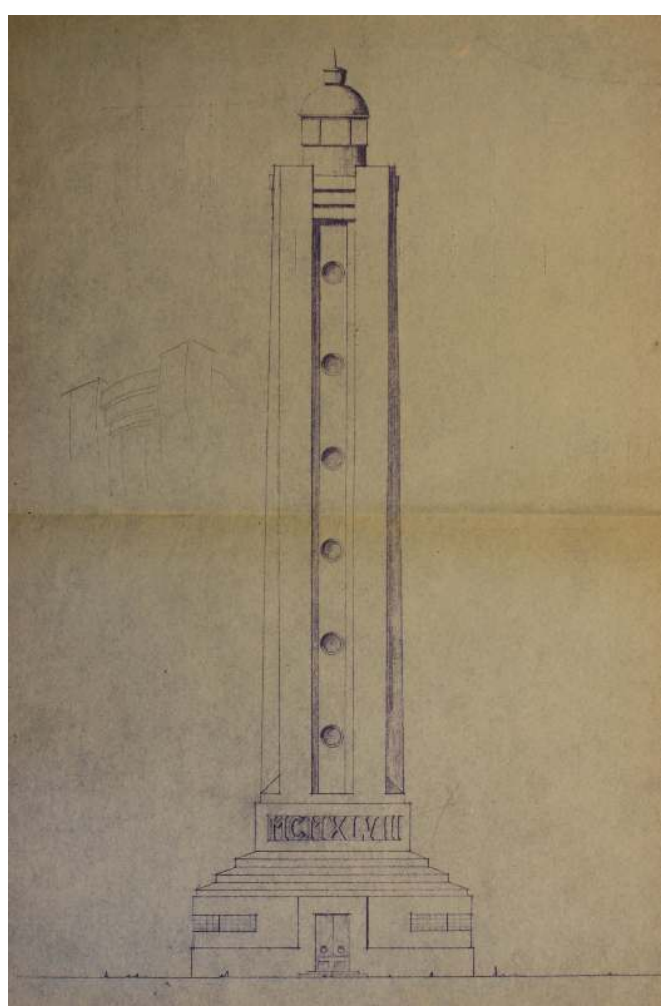

Projet d'élévation de la tour, Maurice Durand, 1946, Arch. nat., F¹4 20134

Cl. Olivier Liardet

Ces trois phares sensiblement contemporains, construits dans les mêmes années où Maurice Durand donnait un visage modernisé à la ville des Sables d'olonne avec le Grand Casino de la promenade du Remblai inauguré en 1951, sont donc les phares de la Reconstruction d'après-guerre. Une quinzaine d'années plus tard, alors qu'il était à la fin de sa carrière, Maurice Durand fut de nouveau chargé d'un chantier de construction pour les Phares et balises, avec le phare de L'Armandèche aux Sables d'Olonne. Dans ce cas-ci, le contexte était différent; il ne s'agissait pas de reconstruire un phare détruit, mais de substituer un ouvrage neuf à un phare ancien devenu obsolète.

11 Auparavant, et depuis le XVIII siècle, le chenal de l'entrée du port, entre le rivage sableux des Sables d'Olonne et le plateau rocheux de La Chaume, était balisé par un feu installé sur la tour de l'ancien château d'Arundel remontant pour l'essentiel au XIVe siècle. En 1965, la politique d'extension de l'urbanisation de la commune des Sables d'Olonne, en faisant le choix d'une densification de l'immobilier par des immeubles collectifs de grande hauteur, rendait nécessaire la construction d'un phare sur le rivage en remplacement du feu d'Arundel masqué par les immeubles de plus de trois étages. Dans sa délibération du 30 juillet, le Conseil municipal constatait qu'il était techniquement impossible d'exhausser la tour d'Arundel de plus de dix-huit mètres pour que le feu fût visible de la mer. En accord avec le ministère de l'Équipement, la commune décidait alors la construction d'un phare sur un terrain communal situé sur le rivage au nord de l'entrée du port, la Ville cédant une superficie d'environ cinq cent $\mathrm{m}^{2}$ au service des Phares et balises, et prenant à sa charge le coût de la construction, estimé «minime en regard des intérêts financiers engagés par les promoteurs pour les opérations de construction en instance et à venir » et qui pourrait être amorti par une redevance payée 
par les promoteurs en fonction du coefficient d'occultation provoquée par les constructions nouvelles.

Le projet de nouveau phare, sur le site de l'Armandèche, fut approuvé par la Grande Commission nautique le 10 décembre $1965^{\circ}$. Le rapport de l'ingénieur en chef présente ainsi le projet : un nouveau phare d'atterrissage d'une puissance égale à celle du Grouin du Cou à La Tranche-sur-Mer, le feu d'Arundel maintenu à sa valeur actuelle; le feu des Barges transformé en feu rouge signalant un danger. Le phare de l'Armandèche sera élevé sur un emplacement sur terrain rocheux entre la laisse des plus hautes mers et la route de corniche envisagée par la municipalité. Le rapport précise : «il est nécessaire de doter la tour de lignes modernes, s'intégrant dans l'unité architecturale de ce secteur $»^{10}$. Le projet fut donc élaboré par le service des Phares et balises en collaboration avec Maurice Durand, « déjà auteur du Grand phare électrique de l'île d'Yeu ». L'édification du nouveau phare fut autorisée par le ministère de l'Équipement le 13 juillet 1966. Le phare fut construit selon le projet établi le 19 août 1966 par l'ingénieur des travaux publics de l'État Bounolleau d'après les esquisses données par Maurice Durand et mis en service le 15 octobre 1968.

III. 6 : Île d'Yeu, phare de La Petite-Foule, soubassement de la tour

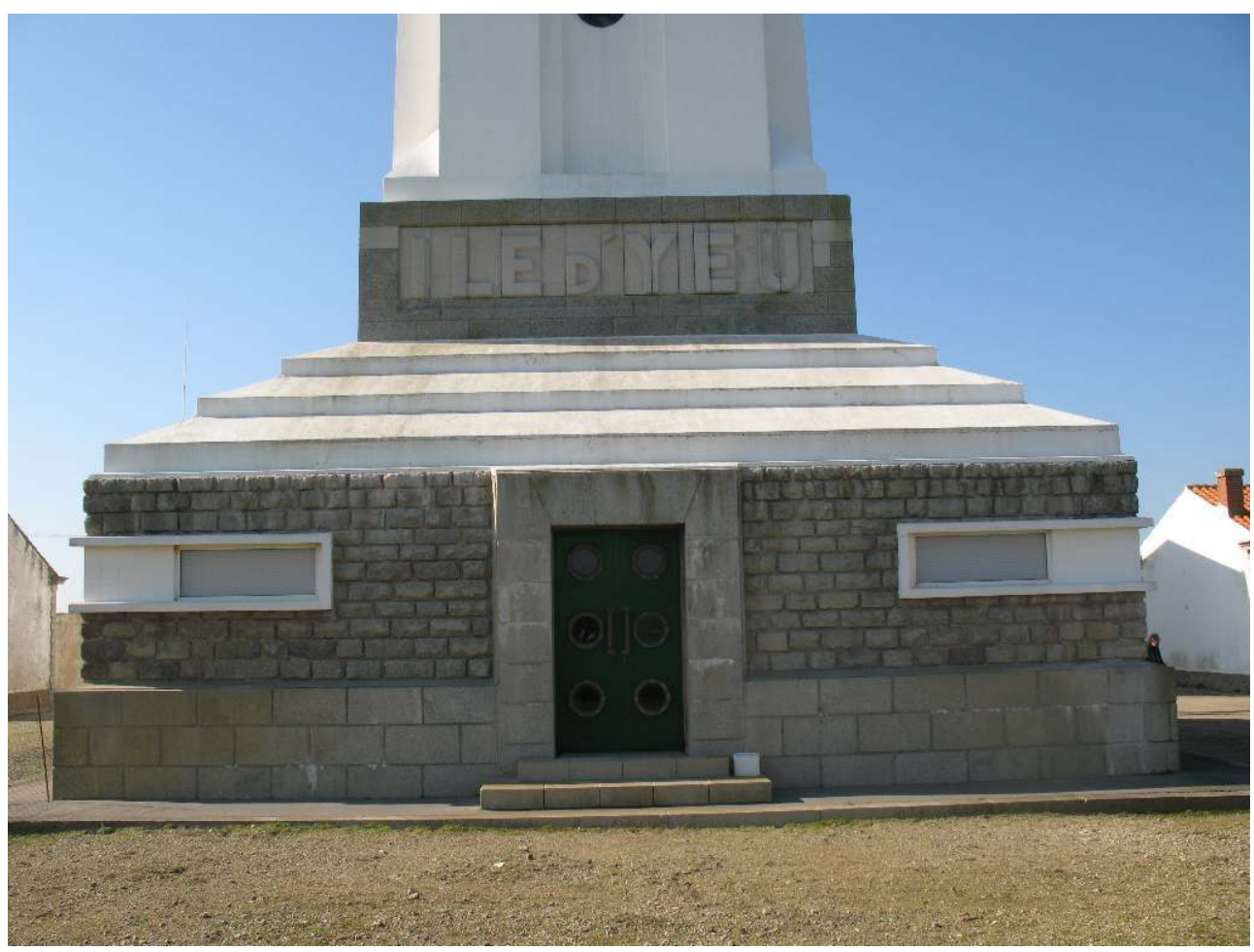

Cl. Alain Delaval

\section{Expérimentations stylistiques}

Les deux grands phares construits sur les dessins de Maurice Durand, le phare de La Petite-Foule à l'île d'Yeu et le phare de L'Armandèche aux Sables d'Olonne, sont très semblables dans leur conception d'ensemble: une tour contenant l'escalier d'accès à la lanterne. Mais l'un et l'autre témoignent de l'évolution du style moderne de Durand au 
cours de la deuxième moitié du siècle. Le phare de La Petite-Foule, tour d'une hauteur de $37,5 \mathrm{~m}$ à section carrée, en maçonnerie de béton lisse sur soubassement pyramidal en maçonnerie de pierres apparentes, fait encore référence aux canons classiques de la tourphare, et aux matériaux traditionnels; l'appareil rustique du soubassement aux percements pliés sur les angles lui donne un aspect à la fois militaire de blockhaus et de tombeau antique avec sa couverture en dalles pyramidante.

Le phare de L'Armandèche, tour en béton armé à section hexadécimale d'une hauteur de $39 \mathrm{~m}$, réalisée avec la technique des coffrages glissants, percé sur les trois faces d'une longue claustra de pavés de verre éclairant l'escalier intérieur, s'il reprend les lignes imposées de la tour-phare, rompt avec le plan habituel carré, circulaire ou octogonal. Le type de percement éclairant l'intérieur rompt avec l'étagement traditionnellement appliqué à l'architecture des phares : là où, à l'île d'Yeu, il éclairait la cage d'escalier par une superposition de hublots, clin-d'œil au style "paquebot» des années 50, à L'Armandèche, il ouvre toute la hauteur du phare par une monumentale fente de verre.

\section{7 : Les Sables-d'Olonne, phare de L'Armandèche}

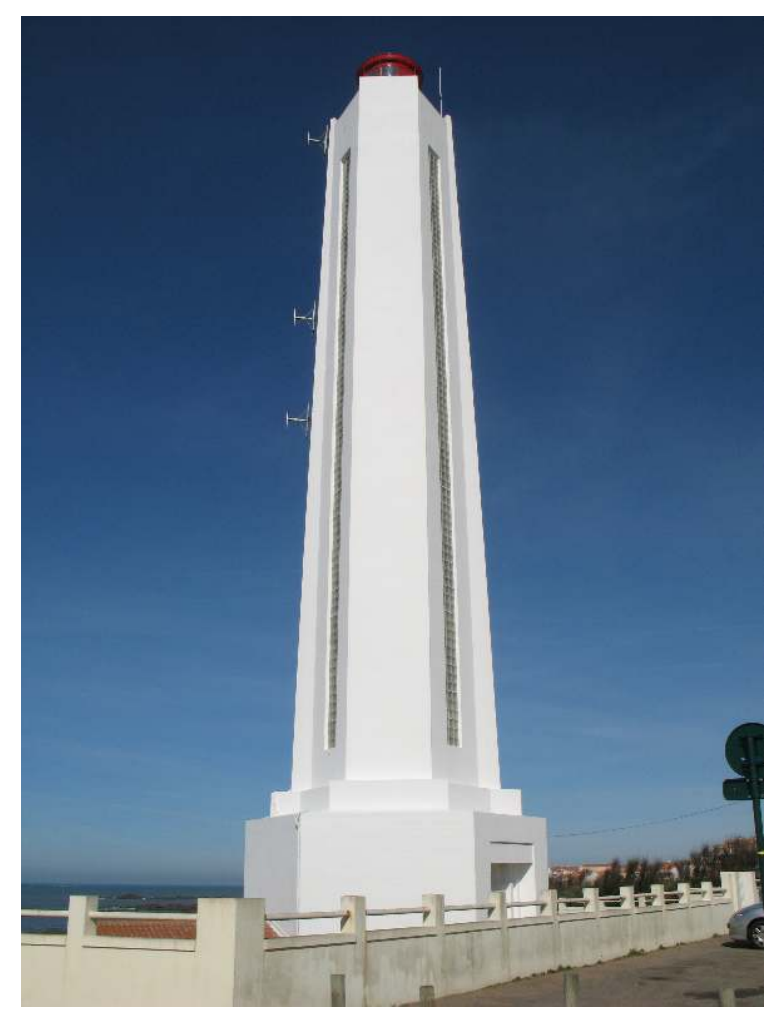

Cl. Alain Delaval

Dernier grand phare construit en France, le phare de L'Armandèche est donc en même temps un manifeste de l'architecture contemporaine; les phares de la reconstruction d'après-guerre n'avaient de moderne que leurs lignes et leur silhouette; le phare de L'Armandèche est plus novateur : il met en œuvre les techniques les plus avancées de la construction en béton armé et propose pour le traitement formel une esthétique de la verticalité lisse, soulignée par les longues claustras de pavés de verre qui éclairent la cage d'escalier sur toute la hauteur de la tour, et un plan hexagonal cantonné de contreforts. Il réalise ainsi la synthèse harmonieuse entre la typologie traditionnelle du phare-tour et l'esthétique moderne des surfaces lisses renonçant à toute interruption factice et 
bannissant tout décor architectural, ne portant pas même un discret relief représentant l'emblème des Phares et Balises. Le phare de L'Armandèche, pour les marins, est un ESM ; pour le spectateur ordinaire, il est une sculpture abstraite.

\section{NOTES}

1. Voir l'article supra sur les concours de phares à l'École des Beaux-arts.

2. Arch. nat., $\mathrm{AJ}^{52} 420$.

3. « Architecture », plaquette illustrée consacrée à Maurice Durand, Strasbourg, EDARI éd., 1932.

4. Arch. dép. Vendée, 2177 W 229, lettre de l'ingénieur ordinaire Renolleau à Maurice Durand du 2 mai 1946, l'invitant à présenter sans tarder les avant-projets de phares «correspondant aux esquisses que vous m'avez soumises au début de février dernier ».

5. Arch. dép. Vendée, 2177 W 221.

6. Arch. dép. Vendée, 2177 W 225 b et 229.

7. Arch. dép. Vendée, 2177 W 223.

8. Arch. dép. Vendée, 2177 W 221.

9. Arch. dép. Vendée, 2177 W 225.

10. Arch. dép. Vendée, 2177 W 224; rapport du 17 janvier 1966.

\section{RÉSUMÉS}

Maurice Durand, (1884-1978), architecte vendéen né aux Sables-d'Olonne en 1884, élève de l'École des Beaux-Arts de Paris, a fait toute sa carrière dans sa ville natale et dans le département de la Vendée dont il fut architecte départemental. Son activité d'architecte s'est exercée essentiellement sur la construction de demeures bourgeoises en bord de mer aux Sablesd'Olonne, ainsi que quelques édifices publics dans sa ville natale dont il fut l'architecte municipal. $\mathrm{Au}$ lendemain de la deuxième guerre mondiale, il fut chargé par le service des Phares et balises de la reconstruction de plusieurs phares détruits par l'occupant Allemand. A la fin de sa carrière, il fut de nouveau appelé à construire le nouveau phare de l'Armandèche aux Sables-d'olonne, construit en 1968.

Maurice Durand (1884-1978), who trained briefly at the École des Beaux-Arts in Paris, spent his entire career working in his birth town of Les Sables-d'Olonne and ultimately succeeded to the position of departmental architect for the Vendée in 1937. He worked primarily for the local bourgeoisie, designing homes on the Atlantic coast and some public buildings in Les Sablesd'Olonne, where he was appointed municipal architect in 1921. After to the Second World War, Durand was commissioned by the Department of Lighthouses and Beacons to rebuild several lighthouses destroyed during the German occupation. At the end of his career, Durand was called 
upon again to design the new lighthouse at Armandèche in the port of Sables-d'Olonne, the last monumental lighthouse of its kind to be built in France, completed in 1968.

Der Architekt Maurice Durand aus der Vendée wurde 1884 in Sables-d'Olonne geboren. Nach seiner Ausbildung an der Pariser École des Beaux-arts verlief seine ganze Karriere in seiner Heimatstadt und im Département Vendée, für welches er zuständiger Architekt wurde. Seine berufliche Tätigkeit als Architekt bezog sich hauptsächlich auf den Bau bürgerlicher Wohnhäuser am Meeresufer in Sables-d'Olonne, so wie auf einige öffentliche Bauten in seiner Heimatstadt, für welche er auch zuständiger Architekt wurde. Unmittelbar nach dem zweiten Weltkrieg wurde er mit dem Wiederaufbau mehrerer durch die deutsche Besatzung zerstörter Leuchttürme beauftragt. Zum Schluss seiner Karriere bekam er noch den Auftrag für die Errichtung des 1968 gebauten Leuchtturms der Armandèche in Les Sables-d'Olonne.

\section{AUTEUR}

\section{ALAIN DELAVAL}

Alain Delaval est chargé d'études documentaires principal à la conservation régionale des Monuments historiques de Nantes, à la direction régionale des Affaires culturelles des Pays de Loire, chargé de la protection au titre des Monuments historiques. Titulaire d'un DEA d'histoire de l'Art (Université de paris I). Auteur d'une monographie du Théâtre Graslin à Nantes (2004), et contributeur de plusieurs ouvrages collectifs, notamment sur l'architecture et l'urbanisme de l'époque des Lumières et de la période napoléonienne, ainsi que sur l'architecture des charpentes anciennes. Membre du Groupe Histoire de l'Architecture et Mentalités Urbaines (GHAMU) fondé par Daniel Rabreau ; membre de la Société Française d'Archéologie et de la Société Archéologique et historique de Nantes. 